\title{
HMM-BASED FRAMEWORK TO MEASURE THE VISUAL FIDELITY OF TONE MAPPING OPERATORS
}

\author{
Waqas Ellahi, Toinon Vigier, Patrick Le Callet \\ firstname.name@univ-nantes.fr \\ LS2N UMR CNRS 6003, Université de Nantes \\ Nantes, France
}

\begin{abstract}
Recent advancements in the image capturing techniques and post processing software generate High Dynamic Range (HDR) images. Such images retain maximum information of the scene by capturing more realistic visual contents, which are often missed in traditional image capturing techniques. In this regard, tone mapping operators (TMOs) play a significant role in displaying HDR image contents on a traditional Low Dynamic Range (LDR) display. These operators tend to introduce artifacts in the original HDR image to change its brightness and contrast in such a way that it can destroy the important textures and information of the image. The assessment of these TMOs is a challenging topic to select best the best technique considering different perceptual and quality dimensions. In this paper, we propose to compare TMOs through their impact on visual behavior in comparison with HDR condition. This study is the first of its kind to utilize hidden markov model (HMM) as a similarity measure to evaluate perceived quality of TMO. The findings suggest that the proposed HMM-based method which emphasizes on temporal information produce better evaluation metric than the traditional approaches which are based only on visual spatial information.
\end{abstract}

Index Terms - scanpath, eye movements, visual attention, hidden markov model, tone mapping operators

\section{INTRODUCTION AND BACKGROUND}

High Dynamic Range (HDR) imaging has gained significant attention in recent years both in industry and academia. The is mainly due to the fact that HDR images are visually more realistic and appealing as they represent a larger dynamic range of the visual content present in the real world. The HDR technologies are arguably considered a major development in display technologies since the transition from black and white to color displays $[1,2]$. However, most of the common imaging devices are only capable to work only with standard dynamic range (SDR) images, leading to a loss of luminance and contrast.
When the dynamic range is lower than the one of the content, the source image is processed with a tone mapping operator (TMO) to compress the dynamic range before rendering. The goal of this technique is to reproduce HDR content on SDR display while preserving details, contrasts and quality. As a consequence, several techniques have been developed in the past utilizing simple global operations such as linear scaling and clipping [3, 4] to more local advance operators that exploit properties of the human visual system $[5,6,7,8,9]$. TMOs can be also perceptually optimized using visual saliency and visual attention to locally adjust the contrast, preserve details and chromoticity [10]. The quality evaluation of tone mapping operators is a challenging topic due to the consideration of multi- perceptual dimensions such as naturalness, colorfulness, brightness, fidelity, and artistic intent. Several studies have assessed these dimensions through subjective experiments $[11,12,13]$ and different objective metrics for HDR content have been proposed [14, 15, 16].

The use of eye tracking experiment is proven to be a good methodology in measuring visual fidelity. Indeed, Narwaria et al. [11] showed that tone mapping operators modify visual attention by comparing the similarity between saliency maps (computed from eye data obtained during the visualisation of HDR and tone-mapped images). The current study performs the comparison of saliency maps and investigates their impact on pre- and post tone mapping. The findings suggest that some salient areas in HDR image are not seen after tone-mapping (and vice versa). However, the dynamic aspect of visual behavior, e.g. scanpaths, is not considered with saliency-based framework. Indeed, temporal aspect in visual attention can reveal the way a content is visually perceived, semantically processed and qualitatively assessed.

Scanpath modeling is a challenging task which has been more recently raised with new analytical tools to compare visual behaviour of different conditions or populations. To cater this issue, probability based approaches are developed by assuming that eye movement parameters are random variables generated by underlying stochastic processes. The set of gaze positions is modeled as bivariate gaussian distribution and hidden markov models (HMMs) are utilized to introduce 
the temporal component of gaze behavior $[17,18,19]$. This in turn will determine the percentage of transitions from one ROI to another ROI. These techniques have been used for the study of scanpath in face recognition [17], the classification of scanpath according to different visual task [19] or different population with visual field losses [18].

The main contribution of the paper is a new HMM based similarly measure in order to evaluate the quality of HDR and TMO generated scanpaths. This paper is structured as follows. First, we explain HMMs in the context of gaze behavior modeling. Then, we illustrate the application of this technique for TMOs bencharmarking. Finally, we conclude our method in the last section.

\section{HMM TOOLS FOR THE MEASURE OF VISUAL SCANPATH SIMILARITY}

\subsection{HMM used as eye movement modeling}

The proposed work utilizes HMMs as a statistical approach to produce the sequence of observation through the set of stochastic process. These observed sequences are then predicted by estimating the transition among different states. it is a data-driven technique and integrates influences stemming from bottom-up mechanisms,top-down mechanisms, and viewing biases into a single model [19]. In this work, the eye position is observed using estimated ROI of the image which is set as the hidden state of HMM. Following the technique used in Chuk et al. [17], the transition matrix represents the saccadic movements of the participant. The emission densities (the distribution of fixation points in each ROI) are modeled as two-dimensional Gaussian distributions. The initial state of the model, i.e., the probability distribution of the first fixation, is modeled with the prior values. The parameter $\mathrm{K}$ which represents the number of ROI in context of scanpath must be selected according to the gaze data instead of setting the parameter with prior information.

The variational approach to Bayesian inference allows simultaneous estimation of complexity of model and its parameters [20], which automates the process of finding number of states (K) in HMM model [17]. An HMM is trained on eye movement data which enables to compare and visualize the gaze behavior of different groups of observers, in different experimental conditions. The individual HMMs of the similar gaze pattern behaviour is clustered by VHEM [21, 22] into joint HMM. For each cluster, the VHEM algorithm produced a representative HMM, which summarizes the common ROIs and transitions in the cluster.

\subsection{Measure of similarity}

In the proposed work, similarity is evaluated using sequence of steps. The steps involved in this process are presented in Fig. 1. Initially, each of the observers eye movement is modeled using HMM for one condition (C1). The HMM of each

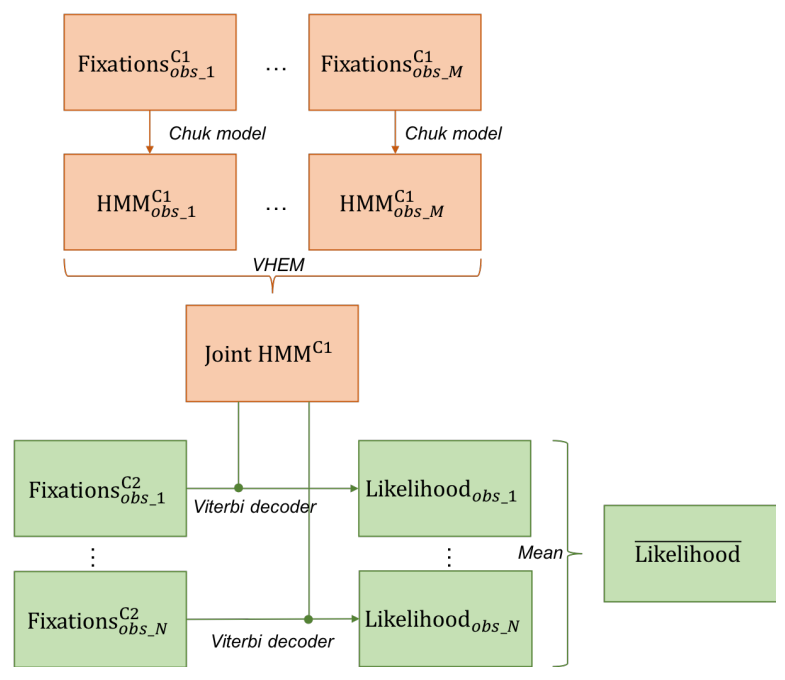

Fig. 1: Scanpath similarity evaluation framework.

observer is then clustered into single Joint HMM by using VHEM. The similarity between the scanpath (i.e. sequence of fixations) of second condition (C2) and the joint clustered is determined by computing the likelihood using Viterbi decorder. The mean likelihood of all observers is considered as a similarity score between the two conditions.

Table 1: Characteristics of subjective experiment

\begin{tabular}{|c|c|c|}
\hline Features & HDR test & TMO test \\
\hline \# of images & 11 & 88 \\
\hline Image resolution & \multicolumn{2}{|c|}{$760 \times 900$ to 1620x1080 } \\
\hline Image duration & \multicolumn{2}{|c|}{10 seconds } \\
\hline Distance & \multicolumn{2}{|c|}{3 times of display height } \\
\hline \# of Observers & 37 & 48 \\
\hline Display model & Sim2 HDR47E & TvLogic LVM401 \\
\hline Display resolution & \multicolumn{2}{|c|}{ 1920x1080 pixels } \\
\hline Eyetracker & \multicolumn{2}{|c|}{ SMI Hi-Speed } \\
\hline
\end{tabular}

\section{APPLICATION OF BENCHMARKING TMOS}

\subsection{Dataset}

The publicly available dataset "Eye-Tracking on High dYnamic range iMAges" (ETHyma) [11] is analyzed in this study. The dataset contains 11 HDR images, captured in different environmental conditions, i.e. indoor and outdoor scenes with varying illumination conditions. A total of 88 TMOs images were computed using 8 different TMO methods. These methods are referred in Table 1, column 4-11, where each column presents the output of these TMO methods using proposed and state-of-the-art techniques. The HDR stimuli were displayed on an HDR display (Sim2 HDR47E S $4 \mathrm{~K})$ and the tone mapped contents are presented on on a stan- 
Table 2: Similarity scores for the studied dataset. (Red cells represent similarity scores higher than the baseline, discussed Section 3.3. Results of green cells are discussed in Section 3.4 to highlight the interest of the framework.)

\begin{tabular}{|c|c|c|c|c|c|c|c|c|c|c|}
\hline \multirow[b]{2}{*}{ Images } & \multirow[b]{2}{*}{ Metrics } & \multirow[b]{2}{*}{$\begin{array}{c}\text { Baseline } \\
\text { Likelihood }\end{array}$} & \multicolumn{8}{|c|}{ TMOs algorithms } \\
\hline & & & $\operatorname{ash}[7]$ & chi [5] & dra [3] & dur [6] & ica $[8]$ & linear & rei [9] & tum [4] \\
\hline \multirow{4}{*}{ Apartment } & $\overline{K L D} \downarrow$ & - & 0.1624 & 0.3320 & 0.2391 & 0.3597 & 0.1309 & 0.1412 & 0.1712 & 0.2236 \\
\hline & $\mathrm{HMM} \uparrow$ & -13.34 & -13.53 & -13.60 & -13.61 & -13.63 & -13.51 & -13.41 & -13.45 & -13.47 \\
\hline & Corr $\uparrow$ & - & 0.8684 & 0.7798 & 0.8010 & 0.7440 & 0.8769 & 0.8761 & 0.8843 & 0.8531 \\
\hline & $\mathrm{NSS} \uparrow$ & - & 1.0238 & 0.8137 & 1.1442 & 0.9361 & 1.0092 & 1.1543 & 1.2349 & 1.2286 \\
\hline \multirow{4}{*}{ Atrium_Night } & KLD $\downarrow$ & - & 0.0917 & 0.1528 & 0.1150 & 0.0796 & 0.1541 & 0.1074 & 0.1543 & 0.1261 \\
\hline & $\mathrm{HMM} \uparrow$ & -13.09 & -13.08 & -13.23 & -13.10 & -13.15 & -13.19 & -13.12 & -13.11 & -13.21 \\
\hline & Corr $\uparrow$ & - & 0.8315 & 0.7451 & 0.8321 & 0.8758 & 0.7174 & 0.8616 & 0.7443 & 0.7281 \\
\hline & $\mathrm{NSS} \uparrow$ & - & 0.8512 & 0.7626 & 0.9471 & 0.9414 & 0.7972 & 0.9478 & 0.6961 & 0.6130 \\
\hline \multirow{4}{*}{ Oxford_Church } & KLD $\downarrow$ & - & 0.2331 & 0.3275 & 0.1880 & 0.2333 & 0.1887 & 0.1897 & 0.1086 & 0.1808 \\
\hline & $\mathrm{HMM} \uparrow$ & -12.74 & -12.94 & -13.03 & -13.06 & -13.03 & -13.06 & -12.94 & -12.61 & -12.69 \\
\hline & Corr $\uparrow$ & - & 0.9273 & 0.8663 & 0.9302 & 0.9100 & 0.8833 & 0.9027 & 0.9134 & 0.8882 \\
\hline & $\mathrm{NSS} \uparrow$ & - & 1.2413 & 1.2006 & 1.2647 & 1.0502 & 1.3077 & 1.3967 & 1.6129 & 1.4484 \\
\hline \multirow{4}{*}{ Big_for_Map } & $\overline{K L D} \downarrow$ & - & 0.1996 & 0.1094 & 0.1880 & 0.1340 & 0.1086 & 0.1350 & 0.1092 & 0.2327 \\
\hline & $\mathrm{HMM} \uparrow$ & -13.02 & -13.10 & -13.17 & -13.36 & -13.26 & -13.29 & -13.39 & -13.22 & -13.25 \\
\hline & Corr $\uparrow$ & - & 0.8018 & 0.8709 & 0.7494 & 0.8376 & 0.8793 & 0.8496 & 0.8764 & 0.7378 \\
\hline & $\mathrm{NSS} \uparrow$ & - & 1.1877 & 0.9602 & 0.6817 & 0.8068 & 0.9608 & 0.7904 & 1.1687 & 0.8693 \\
\hline \multirow{4}{*}{ Dani_Belgium } & KLD $\downarrow$ & - & 0.1775 & 0.1278 & 0.2162 & 0.1589 & 0.2152 & 0.2013 & 0.2025 & 0.2288 \\
\hline & $\mathrm{HMM} \uparrow$ & -13.03 & -13.12 & -12.96 & -13.23 & -13.15 & -13.03 & -12.98 & -13.17 & -13.11 \\
\hline & Corr $\uparrow$ & - & 0.7239 & 0.8279 & 0.6537 & 0.8185 & 0.7045 & 0.8241 & 0.7544 & 0.7393 \\
\hline & $\mathrm{NSS} \uparrow$ & - & 0.8347 & 1.1032 & 0.7022 & 1.0347 & 0.8727 & 1.2562 & 0.8626 & 0.7540 \\
\hline \multirow{4}{*}{ lampickaHDR } & $\overline{K L D} \downarrow$ & - & 0.2234 & 0.1485 & 0.1645 & 0.2338 & 0.5603 & 0.1619 & 0.0821 & 0.2884 \\
\hline & $\mathrm{HMM} \uparrow$ & -12.68 & -13.00 & -13.01 & -13.35 & -13.27 & -13.08 & -13.24 & -12.84 & -13.29 \\
\hline & Corr $\uparrow$ & - & 0.9154 & 0.8306 & 0.8322 & 0.6259 & 0.8719 & 0.7600 & 0.9367 & 0.5663 \\
\hline & $\overline{N S S ~ \uparrow ~}$ & - & 1.2041 & 0.9626 & 0.9040 & 0.8113 & 1.0356 & 0.8568 & 1.2953 & 0.7550 \\
\hline \multirow{4}{*}{ memorial_o876 } & KLD $\downarrow$ & - & 0.1125 & 0.1092 & 0.0729 & 0.0801 & 0.0565 & 0.0773 & 0.0551 & 0.1037 \\
\hline & $\mathrm{HMM} \uparrow$ & -12.31 & -12.35 & -12.63 & -12.54 & -12.40 & -12.53 & -12.35 & -12.49 & -12.33 \\
\hline & Corr $\uparrow$ & - & 0.9037 & 0.7787 & 0.9156 & 0.8978 & 0.9362 & 0.9147 & 0.9335 & 0.8886 \\
\hline & $\mathrm{NSS} \uparrow$ & - & 1.2283 & 0.7700 & 1.0032 & 0.9547 & 0.8890 & 1.0938 & 1.0564 & 1.2794 \\
\hline \multirow{4}{*}{ moto } & KLD $\downarrow$ & - & 0.7040 & 0.1932 & 0.1353 & 0.1967 & 0.2049 & 0.2216 & 0.3256 & 0.1876 \\
\hline & $\mathrm{HMM} \uparrow$ & -13.39 & -13.50 & -13.41 & -13.56 & -13.48 & -13.32 & -13.39 & -13.68 & -13.55 \\
\hline & Corr $\uparrow$ & - & 0.8894 & 0.9066 & 0.9048 & 0.8633 & 0.9086 & 0.9014 & 0.8253 & 0.8551 \\
\hline & $\mathrm{NSS} \uparrow$ & - & 1.4345 & 1.5737 & 1.3882 & 1.4694 & 1.5536 & 1.8779 & 1.1869 & 1.3414 \\
\hline \multirow{4}{*}{ forest_path } & KLD $\downarrow$ & - & 0.3322 & 0.4180 & 0.1626 & 0.1235 & 0.1458 & 0.1791 & 0.1679 & 0.1265 \\
\hline & $\mathrm{HMM} \uparrow$ & -13.47 & -13.84 & -13.83 & -13.75 & -13.67 & -13.68 & -13.83 & -13.79 & -13.61 \\
\hline & Corr $\uparrow$ & - & 0.8224 & 0.8475 & 0.9295 & 0.9447 & 0.9461 & 0.8724 & 0.9408 & 0.9475 \\
\hline & $\mathrm{NSS} \uparrow$ & - & 1.3002 & 1.1029 & 1.5537 & 1.3769 & 1.5853 & 1.2878 & 1.6714 & 1.4998 \\
\hline \multirow{4}{*}{ rend02_oC95 } & KLD $\downarrow$ & - & 0.1488 & 0.0989 & 0.1080 & 0.0869 & 0.0978 & 0.0765 & 0.1187 & 0.0716 \\
\hline & $\mathrm{HMM} \uparrow$ & -12.90 & -12.97 & -12.91 & -13.04 & -12.91 & -12.96 & -12.94 & -13.06 & -12.96 \\
\hline & $\operatorname{Corr} \uparrow$ & - & 0.8431 & 0.8617 & 0.8927 & 0.9404 & 0.9078 & 0.9212 & 0.8792 & 0.9263 \\
\hline & $\mathrm{NSS} \uparrow$ & - & 1.0016 & 1.0149 & 1.1647 & 1.4003 & 1.1596 & 1.2397 & 1.0846 & 1.3691 \\
\hline \multirow{4}{*}{ treeUnil } & KLD $\downarrow$ & - & 0.0835 & 0.0963 & 0.1867 & 0.1437 & 0.0637 & 0.1939 & 0.1188 & 0.1965 \\
\hline & $\mathrm{HMM} \uparrow$ & -13.19 & -13.38 & -13.29 & -13.43 & -13.36 & -13.25 & -13.31 & -13.31 & -13.10 \\
\hline & Corr $\uparrow$ & - & 0.9540 & 0.9297 & 0.8172 & 0.8268 & 0.9540 & 0.8527 & 0.9092 & 0.8737 \\
\hline & $\overline{\mathrm{NSS} \uparrow}$ & - & 1.0772 & 1.1153 & 0.8824 & 0.9030 & 1.2082 & 1.1491 & 1.2066 & 1.3509 \\
\hline
\end{tabular}


dard LCD screen. Each image was viewed by the participant during 10 seconds. The eye movements were recorded using SMI Hi-Speed tracker with a frequency of $500 \mathrm{~Hz}$. Thirty seven observers participated in the HDR experiment and 48 did the TMO experiment. The characteristics of this subjective experiment are presented in Table.1.

\subsection{Similarity metrics}

In this section, we present the obtained scores of similarity for our framework compared with state-of-the-art saliency-based metrics: Kullback-Liebler divergence (KLD), 2D correlations (Corr) and Normalized scanpath saliency (NSS) [23]. These metrics are calculated after the computation of fixation maps. These maps are computed by convolving a Gaussian kernel with standard deviation of one degree of visual angle across the observer's fixation locations. The proposed framework is applied with $\mathrm{C} 1$ as the HDR condition compared with the different tone mapping techniques. The HMM model is applied on eye fixations extracted from raw gaze data for each observer during HDR experiment [24]. In addition, each HMM is then clustered into single joint HMM by using VHEM. The likelihood is calculated using Viterbi decoder by passing joint HMM parameters and fixation points recorded during TMOs experiment. The similarity score between HDR and TMOs is estimated by taking mean of likelihood of all observers for each TMOs. In this study, the maximum value of states in HMM, $K$, is set to 7 . The scores of the proposed framework with the ones of all the other similarity / dissimilarity metrics are presented Table 2 .

Table 3: Pearson correlation coefficients (PCC) between similarity metrics. ( $*$ indicates a p-value $<0.05$ and $* *$ indicates a p-value $<0.001)$.

\begin{tabular}{|c|c|c|c|c|}
\hline PCC & KLD & HMM & Corr & NSS \\
\hline KLD & 1.0000 & $-0.3957^{* *}$ & $-0.2755^{*}$ & 0.0581 \\
\hline HMM & $-0.3975^{* *}$ & 1.0000 & 0.1300 & -0.1472 \\
\hline Corr & $-0.2755^{*}$ & 0.1300 & 1.0000 & $0.6811^{* *}$ \\
\hline NSS & 0.0581 & -0.1472 & $0.6811^{* *}$ & 1.0000 \\
\hline
\end{tabular}

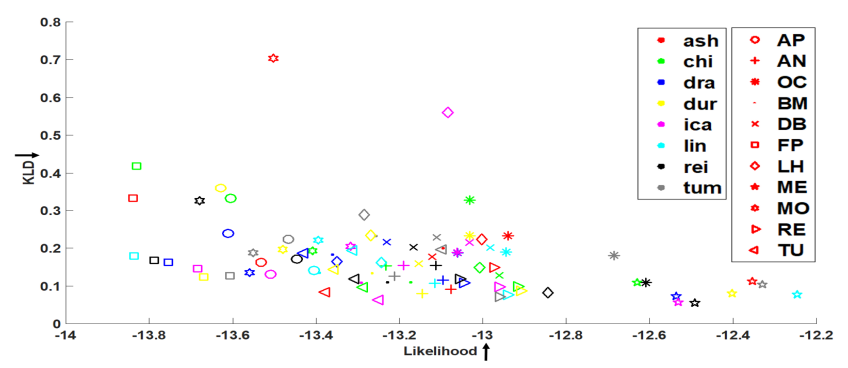

Fig. 2: Relationship between scores of KLD and HMM metrics
In addition, a baseline likelihood score on individual HDR scanpaths is also calculated to validate this method. The baseline likelihood is estimated by providing the fixation points of each observer for HDR condition and joint HMM parameter to the Viterbi decoder. The final score is taking a mean of all likelihood for all observers. This baseline can be interpreted as the similarity score between one observer and a model of all scanpaths for the same condition. It is thus expected that the likelihood score for the TM condition would be lower than the baseline.

\subsection{Validation of the framework}

The similarity metrics are compared between each other through the computation of Pearson's correlation coefficients (PCC) presented Table 3. The proposed framework shows a strong significant negative correlations with KLD metric. The relationship between KLD and HMM likelihood scores is also presented Fig. 2. These results ensure the validity of the proposed framework which is correlated with existing metrics used to measure visual fidelity.

Concerning the baseline, the obtained scores seem to validate the previous assumption except for a few values (highlighted in red in Table 2). These specific cases could be explained by a low inter-observer congruency for HDR baseline condition and further analysis should be done to better understand these outcomes.

\subsection{Benchmarking of TMOs through dynamic visual fi- delity}

The similarity scores detailed in Table 2 show a strong correlation between saliency-based metrics and the proposed HMM-based one, except for some specific tone-mapped contents. The moto and rend02_oC95 are selected as test images to investigate these results through the comparison of the performance between HMM and KLD. The performed analysis of KLD suggests that Drago and Tumblin methods offer the best performance among all for moto and rend02_oC95 images respectively. Whereas with the HMM-based framework, iCAM06 method offers best performance for moto and Chiu method for rend02_oC95 image (see Table 2-highlighted green cells). Saliency maps, HDR joint HMM states and transition matrix as well as scanpaths for TM0 condition are depicted on Fig 3 and 4. We can see that most performant TMOs with our framework seem to lead to more similar saliency maps than the best ones with KLD metric. In addition, the scanpaths for iCAM06 (resp. Chiu) TMO fit better the joint HMM on HDR condition than Drago (resp. Tumblin) TMO. From these observations, we can see that HMM-based framework outperfoms KLD metric and better reveals visual attention similarity for both spatial (i.e salient) and temporal (i.e. scanpath) information.

One limitation of this proposed framework is that HMM based on Chuck methodology perform well with image con- 

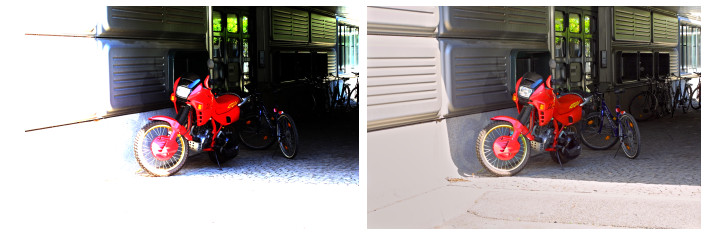

(a) Drago TMO output

(b) iCAM06 TMO output

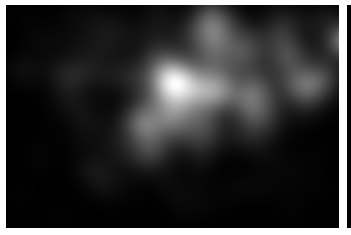

(c) HDR saliency map

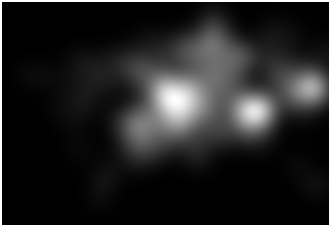

(d) Drago saliency map

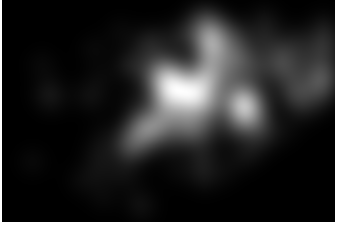

(e) iCAM06 saliency map
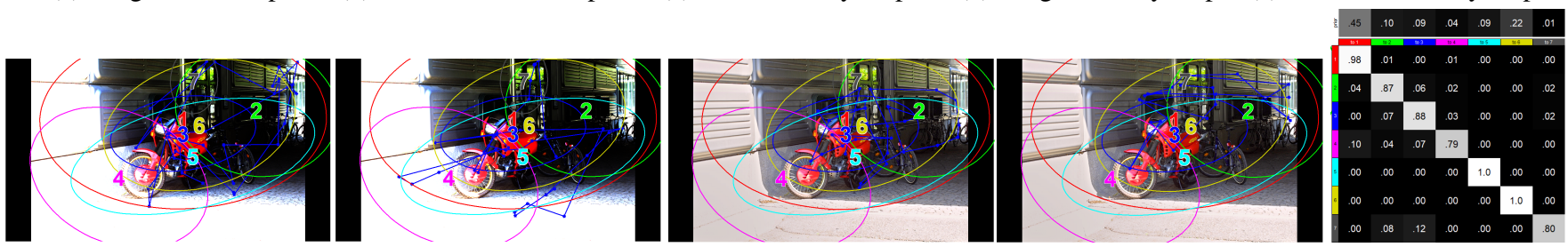

(f) HDR joint HMM clusters with 2 scanpaths on Drago (g) HDR joint HMM clusters with 2 scanpaths on iCAM06 (h) HMM transitions TMO TMO matrix

Fig. 3: Tone-mapped images, saliency maps, scanpaths and HDR joint HMM model for moto content.

tent with clear regions of interest and, thus, low visual attention dispersion. Therefore, future work should be needed to deeper assess the impact of content, inter-observer congruency and HMM meta-parameters as number of states (i.e regions of interest) on the similarity metric performance. Different HMM techniques can be also further tested and compared for similarity evaluation through this framework.

\section{CONCLUSION}

In this paper, an HMM based framework is proposed to measure the similarity of visual behaviour between two conditions of experiment. The framework is then applied and validated for the comparison of visual attention in HDR and tonemapped images. The proposed framework provides promising results due to the capability of HMM to analyse eye data considering both the spatial and sequential aspects. On the contrary, other saliency models do not reflect the dynamic (saccadic) nature of visual scanpaths.

\section{ACKNOWLEDGMENT}

The work in this paper was funded from the European Union's Horizon 2020 research and innovation program under the Marie Sklodowska-Curie grant agreement No 765911, European Training Network on Real Vision project.

\section{REFERENCES}

[1] Ahmet Oğuz Akyüz, Roland Fleming, Bernhard E Riecke, Erik Reinhard, and Heinrich H Bülthoff, "Do hdr displays support ldr content? a psychophysical evaluation," ACM Transactions on Graphics (TOG), vol. 26, no. 3, pp. 38-es, 2007.
[2] F Banterle, A Artusi, K Debattista, and A Chalmers, "Advanced high dynamic range imaging: Theory and practice. isbn: 978-156881-719-4, ak peters," .

[3] Frédéric Drago, Karol Myszkowski, Thomas Annen, and Norishige Chiba, "Adaptive logarithmic mapping for displaying high contrast scenes," in Computer graphics forum. Wiley Online Library, 2003, vol. 22, pp. 419-426.

[4] Jack Tumblin, Jessica K Hodgins, and Brian K Guenter, "Two methods for display of high contrast images," ACM Transactions on Graphics (TOG), vol. 18, no. 1, pp. 56-94, 1999.

[5] Ken Chiu, Michael Herf, Peter Shirley, S Swamy, Changyaw Wang, Kurt Zimmerman, et al., "Spatially nonuniform scaling functions for high contrast images," in Graphics Interface. Canadian Information Processing Society, 1993, pp. 245-245.

[6] Frédo Durand and Julie Dorsey, "Fast bilateral filtering for the display of high-dynamic-range images," in Proceedings of the 29th annual conference on Computer graphics and interactive techniques, 2002, pp. 257-266.

[7] Michael Ashikhmin, "A tone mapping algorithm for high contrast images," in Proceedings of the 13th Eurographics workshop on Rendering. Eurographics Association, 2002, pp. 145-156.

[8] Jiangtao Kuang, Garrett M Johnson, and Mark D Fairchild, "icam06: A refined image appearance model for hdr image rendering," Journal of Visual Communication and Image Representation, vol. 18, no. 5, pp. 406-414, 2007.

[9] Erik Reinhard, Michael Stark, Peter Shirley, and James Ferwerda, "Photographic tone reproduction for digital images," in Proceedings of the 29th annual conference on Computer graphics and interactive techniques, 2002, pp. 267-276. 




(a) Tumblin TMO output

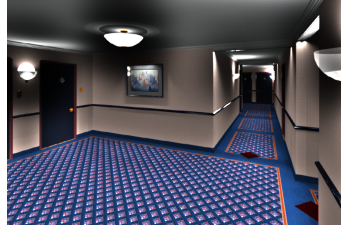

(b) Chiu TMO output

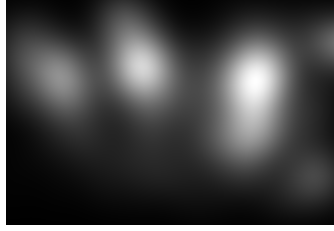

(c) HDR saliency map



(d) Tumblin saliency map

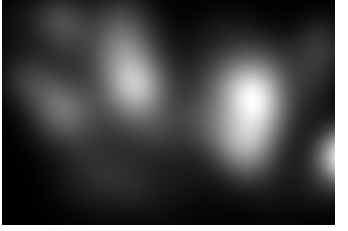

(e) Chiu saliency map
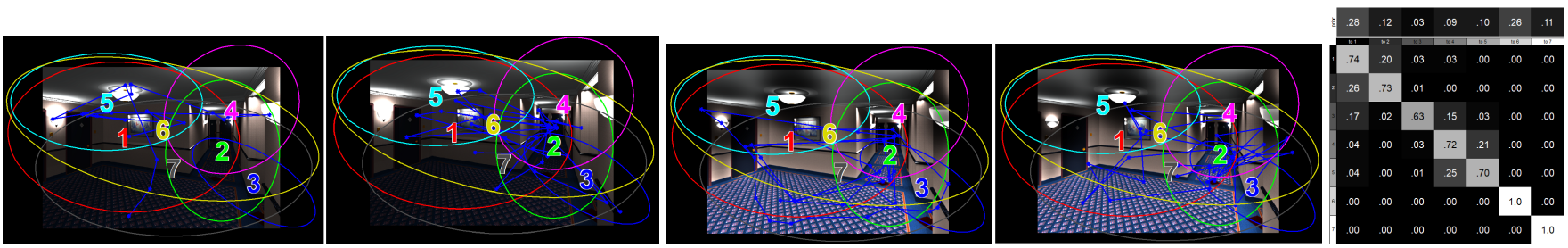

(f) HDR joint HMM clusters with 2 scanpaths on Tumblin (g) HDR joint HMM clusters with 2 scanpaths on Chiu (h) HMM transitions TMO TMO matrix

Fig. 4: Tone-mapped images, saliency maps, scanpaths and HDR joint HMM model for rend02_oC95 content.

[10] Wen-Chieh Lin and Zhi-Cheng Yan, "Attention-based high dynamic range imaging," The Visual Computer, vol. 27, no. 6-8, pp. 717, 2011.

[11] Manish Narwaria, Matthieu Perreira Da Silva, Patrick Le Callet, and Romuald Pépion, "Effect of tone mapping operators on visual attention deployment," in $A p$ plications of Digital Image Processing XXXV. International Society for Optics and Photonics, 2012, vol. 8499, p. $84990 \mathrm{G}$.

[12] Martin Čadík, Michael Wimmer, Laszlo Neumann, and Alessandro Artusi, "Evaluation of hdr tone mapping methods using essential perceptual attributes," Computers \& Graphics, vol. 32, no. 3, pp. 330-349, 2008.

[13] Josselin Petit and Rafał K Mantiuk, "Assessment of video tone-mapping: Are cameras' s-shaped tonecurves good enough?," Journal of Visual Communication and Image Representation, vol. 24, no. 7, pp. 10201030, 2013.

[14] Lukáš Krasula, Karel Fliegel, Patrick Le Callet, and Miloš Klíma, “Objective evaluation of naturalness, contrast, and colorfulness of tone-mapped images," in $A p$ plications of Digital Image Processing XXXVII. International Society for Optics and Photonics, 2014, vol. 9217 , p. 92172D.

[15] Hojatollah Yeganeh and Zhou Wang, "Objective quality assessment of tone-mapped images," IEEE Transactions on Image Processing, vol. 22, no. 2, pp. 657-667, 2012.

[16] Miguel Granados, Tunc Ozan Aydin, J Rafael Tena, Jean-Francois Lalonde, and Christian Theobalt, "Contrast-use metrics for tone mapping images," in 2015 IEEE International Conference on Computational Photography (ICCP). IEEE, 2015, pp. 1-8.

[17] Tim Chuk, Antoni B Chan, and Janet H Hsiao, "Understanding eye movements in face recognition using hid- den markov models," Journal of vision, vol. 14, no. 11, pp. 8-8, 2014.

[18] Erwan Joël David, Pierre Lebranchu, Matthieu Perreira Da Silva, and Patrick Le Callet, "Predicting artificial visual field losses: a gaze-based inference study," Journal of Vision, vol. 19, no. 14, pp. 22-22, 2019.

[19] Antoine Coutrot, Janet H Hsiao, and Antoni B Chan, "Scanpath modeling and classification with hidden markov models," Behavior research methods, vol. 50, no. 1, pp. 362-379, 2018.

[20] Clare A McGrory and DM Titterington, "Variational bayesian analysis for hidden markov models," Australian \& New Zealand Journal of Statistics, vol. 51, no. 2, pp. 227-244, 2009.

[21] Emanuele Coviello, Gert R Lanckriet, and Antoni B Chan, "The variational hierarchical em algorithm for clustering hidden markov models," in Advances in neural information processing systems, 2012, pp. 404-412.

[22] Emanuele Coviello, Antoni B Chan, and Gert RG Lanckriet, "Clustering hidden markov models with variational hem," The Journal of Machine Learning Research, vol. 15, no. 1, pp. 697-747, 2014.

[23] Mohsen Emami and Lawrence L Hoberock, "Selection of a best metric and evaluation of bottom-up visual saliency models," Image and Vision Computing, vol. 31, no. 10, pp. 796-808, 2013.

[24] Asim H Dar, Adina S Wagner, and Michael Hanke, "Remodnav: Robust eye movement detection for natural viewing," BioRxiv, p. 619254, 2020. 